\title{
ON THE VARIABILITY OF SUPERGIANTS OF TYPES B-G
}

\author{
A. MAEDER and F. RUFENER \\ Geneva Observatory, Switzerland
} \begin{abstract}
in Table I.
TABLE I

Comparison of supergiants with normal MS stars

\begin{tabular}{lllll}
\hline $\begin{array}{l}\text { Luminosity } \\
\text { class }\end{array}$ & $N$ & $\Delta m_{r}$ & $\Delta C$ & $p$ \\
\hline la & 22 & $0^{\mathrm{m}} 063$ & $0^{\mathrm{m}} \cdot 024$ & 9 \\
Iab-Ib & 35 & $0^{\mathrm{m}} \cdot 045$ & $0^{\mathrm{m}} \cdot 021$ & 6 \\
II & 19 & $0^{\mathrm{m}} \cdot 027$ & $0^{\mathrm{m}} \cdot 017$ & 5 \\
\hline As a comparison: & $0^{\mathrm{m}} \cdot 023$ & $0^{\mathrm{m}} \cdot 018$ & 6 \\
\multicolumn{2}{l}{ Normal MS stars } & & & \\
\hline
\end{tabular}
\end{abstract}

Abstract. The variability of the B-G supergiants is studied here with a set of 500 observations made for 76 stars. In addition to the discussion on the variability in colour indices (Maeder and Rufener, 1972), we also include the analysis of the apparent magnitudes. The observations, made randomly in time over several years, are selected from the new Catalogue (Rufener, 1974) of stars measured in the Geneva Observatory system. Some results on the luminosity dependence of the variations are presented

$N$ is the number of stars observed in each luminosity class. $\Delta m_{v}$ and $\Delta C$ are the amplitudes of the variations in $V$ magnitude and colour indices. These amplitudes are defined as the range containing $90 \%$ of the individual measurements for a given star under the hypothesis of a normal distribution of the deviations to the mean. The quantity $p$ is the mean number of individual measurements for each star. Table I clearly shows that the amplitude of the variations is increasing with increasing luminosity. From the study of the distribution of the amplitudes in each class, we may also infer that all supergiants of class Ia are variable in light. The stars of class II are essentially non variable for our threshold of sensitivity to variations. The stars of class Iab and $\mathrm{Ib}$ are intermediate.

The spectrum variations of the supergiants have been studied by several authors. Many spectral features, such as blue displaced circumstellar lines, reveal the existence of mass loss for these stars. However, indications of pulsation-type motions, besides the mass loss effects, have been given by Abt (1957) and especially by Chentsov and Snezhko (1971). From Figure 1, where luminosity is plotted vs period (or cycles) collected in the literature, we may draw further arguments in favour of the existence of some pulsation mechanism in supergiants. Figure 1 shows that the position of stars in the period-luminosity diagram is (a) spectral type dependent and (b) rather parallel 


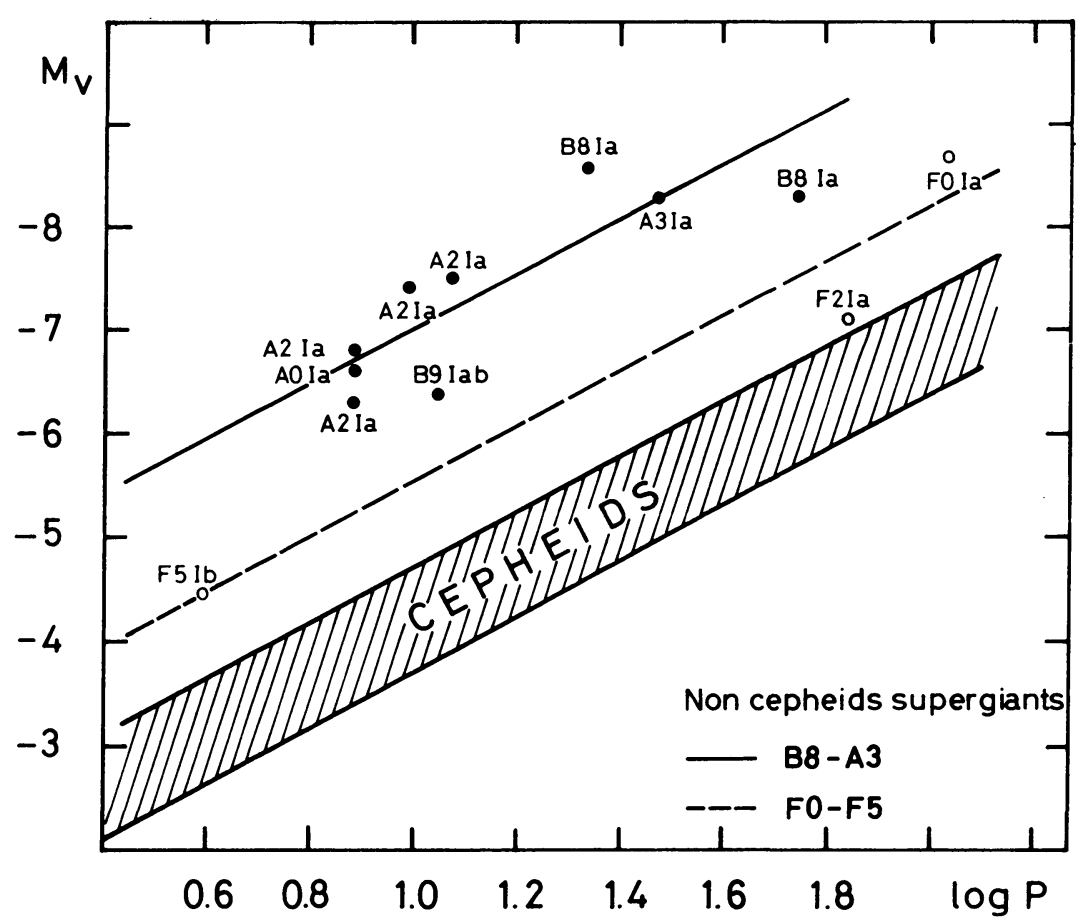

Fig. 1. Position of some supergiants in the period-luminosity diagram.

to the period-luminosity relation for Cepheids, as given by Sandage and Tammann (1968). These two facts may well be explained in the following way. If the variations are due to some oscillatory motion, one has the relation: $P \sim Q \varrho^{-1 / 2}$ which gives, along with the mass-luminosity relation and the definition of $T_{\text {eff }}: \log P=\log Q-$ $-3 \log T_{\text {eff }}-0.24 M_{\text {bol }}+C . Q$ characterizes both the stellar structure and the oscillatory motion. The above relation accounts reasonably well for the posision of the early supergiants relatively to the Cepheids in the P-L diagram and for the slope of this relation. The similarity of the $Q$-value for B-G supergiants and Cepheids is also well supported by the study of Figure 1. This suggests that the supergiants are subject to radial pulsation in the fundamental mode.

These facts (cycle-luminosity-amplitude relations) give some indications on the association of pulsation and mass loss in the supergiant star located in the HR diagram to the left of the instability strip of the Cepheids.

\section{References}

Abt, H. A.: 1957, Astrophys. J. 126, 138.

Chentsov, E. L. and Snezhko, L. I.: 1971, in M. Hack (ed.), Supergiant Stars, 3rd Colloquium on Astrophysics held in Trieste, Osservatorio Astronomico, Trieste, Italy, p. 51.

Maeder, A. and Rufener, F.: 1972, Astron. Astrophys. 20, 437.

Rufener, F.: 1974, Astron. Astrophys. Suppl. (in preparation).

Sandage, A. and Tammann, G. A.: 1968, Astrophys. J. 151, 531. 


\section{DISCUSSION}

Rodgers: Are there significantly non-periodic variations that are in excess of the errors that you have quoted?

Maeder: We have undertaken a set of long time base measurements of supergiants near the double cluster $h$ and $\chi$ Persei, and it is measurements such as these that will answer your question.

Breger: The variability of HR 7308, an F supergiant, confirms Dr Maeder's comments. More than 100 observations show a fairly constant amplitude with a semi-period. The variations do not appear irregular, except for the slight difficulty with the period.

Underhill: You mentioned observing the variations of Be stars as well as the supergiants. Do you think the variations are of the same type?

Maeder: No, clearly no. 